\title{
G

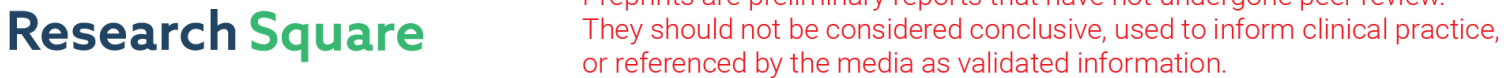

\section{Long-term Specific IgG Response to SARS-CoV-2 Nucleocapsid Protein in Recovered COVID-19 Patients}

\author{
Jira Chansaenroj
}

Center of Excellence in Clinical Virology, Faculty of Medicine, Chulalongkorn University Ritthideach Yorsaeng

Center of Excellence in Clinical Virology, Faculty of Medicine, Chulalongkorn University

\section{Nawarat Posuwan}

Center of Excellence in Clinical Virology, Faculty of Medicine, Chulalongkorn University Jiratchaya Puenpa

Center of Excellence in Clinical Virology, Faculty of Medicine, Chulalongkorn University

\section{Nasamon Wanlapakorn}

Center of Excellence in Clinical Virology, Faculty of Medicine, Chulalongkorn University

\section{Natthinee Sudhinaraset}

Center of Excellence in Clinical Virology, Faculty of Medicine, Chulalongkorn University

\section{Manit Sripramote}

Medical Service Department, Bangkok Metropolitan Administration

\section{Piti Chalongviriyalert}

Medical Service Department, Bangkok Metropolitan Administration

\section{Supunee Jirajariyavej}

Taksin Hospital, Medical Service Department, Bangkok Metropolitan Administration

Phatharaporn Kiatpanabhikul

Charoenkrung Pracharak Hospital, Medical Service Department, Bangkok Metropolitan Administration Jatuporn Saiyarin

Klang General Hospital, Medical Service Department, Bangkok Metropolitan Administration

\section{Chulikorn Soudon}

Sirindhorn Hospital, Medical Service Department, Bangkok Metropolitan Administration

\section{Orawan Thienfaidee}

Ratchaphiphat Hospital, Medical Service Department, Bangkok Metropolitan Administration

\section{Thitisan Palakawong Na Ayuthaya}

Public Health Center 28, Health Department, Bangkok Metropolitan Administration

\section{Chantapat Brukesawan}

Public Health Center 26, Health Department, Bangkok Metropolitan Administration 
Center of Excellence in Clinical Virology, Faculty of Medicine, Chulalongkorn University

\section{Duangnapa Intharasongkroh}

National Blood Center, Thai Red Cross Society

\section{Dootchai Chaiwanichsiri}

National Blood Center, Thai Red Cross Society

\section{Mila Issarasongkhram}

Institute for Urban Disease Control and Prevention, Department of Disease Control, Ministry of Public Health

\section{Rungrueng Kitphati}

Institute for Urban Disease Control and Prevention, Department of Disease Control, Ministry of Public Health

\section{Anek Mungaomklang}

Institute for Urban Disease Control and Prevention, Department of Disease Control, Ministry of Public Health

\section{Pijaya Nagavajara}

Office of the Permanent Secretary for the Bangkok Metropolitan Administration

\section{Yong Poovorawan ( $\nabla$ yong.p@chula.ac.th )}

Center of Excellence in Clinical Virology, Faculty of Medicine, Chulalongkorn University

\section{Research Article}

Keywords: Long-term, SARS-CoV-2, COVID-19, Nucleocapsid antibody, IgG, Recovery

Posted Date: July 1st, 2021

DOI: https://doi.org/10.21203/rs.3.rs-608525/v1

License: (9) This work is licensed under a Creative Commons Attribution 4.0 International License. Read Full License

Version of Record: A version of this preprint was published at Scientific Reports on December 1st, 2021. See the published version at https://doi.org/10.1038/s41598-021-02659-4. 


\section{Abstract}

This study monitored the long-term immune response to severe acute respiratory syndrome coronavirus (SARS-CoV)-2 infection in patients who had recovered from coronavirus disease (COVID)-19. Antinucleocapsid immunoglobulin $\mathrm{G}$ (anti-N IgG) titer in serum samples collected at a single $(\mathrm{N}=302)$ or multiple time points ( $\mathrm{N}=229)$ 3-12 months after COVID-19 symptom onset or SARS-CoV-2 detection in respiratory specimens was measured by semiquantitative chemiluminescent microparticle immunoassay. The 531 patients (966 specimens) were classified according to the presence or absence of pneumonia symptoms. Anti-N IgG was detected in $87.5 \%$ of patients (328/375) at 3 months, $38.6 \%(93 / 241)$ at 6 months, $23.7 \%(49 / 207)$ at 9 months, and $26.6 \%(38 / 143)$ at 12 months. The anti-N IgG seropositivity rate was significantly lower at 6,9 , and 12 months than at 3 months $(P<0.01)$ and was higher in the pneumonia group than in the non-pneumonia/asymptomatic group at 6 months $(P<0.01), 9$ months $(P=0.04)$, and 12 months $(P=0.04)$. The rate started to decline $6-12$ months after symptom onset. Anti-N IgG sample/cutoff index was positively correlated with age $(r=0.192, P<0.01)$ but negatively correlated with interval between symptom onset and blood sampling $(r=-0.567, P<0.01)$. These findings can guide vaccine strategies in recovered COVID-19 patients.

\section{Introduction}

Coronavirus disease 2019 (COVID-19) has a broad spectrum clinical manifestations including asymptomatic, mild, severe, critical, and even fatal [1,2]. A large proportion of infected individuals are asymptomatic or have mild symptoms but are still contagious [1]. The elderly and individuals with chronic medical conditions are more likely to become severely ill from COVID-19. The severe cases eventually develop acute respiratory distress syndrome, multiple organ failure, pneumonia, and cytokine storm [3]. Diagnostic testing for early identification and isolation of infected persons and timely treatment is the main strategy used to control the spread of COVID-19.

A commonly used diagnostic test for COVID-19 is a reverse-transcription (RT)-PCR assay that detects one or more viral RNA genes reflecting current or recent infection. However, RT-PCR has some limitations: it requires sample processing in a laboratory with specialized reagents, instruments, and well-trained laboratory staff; and the poor quality of nasal or nasopharyngeal swab specimens and RNA instability can lead to false-negative results. Antibody or serologic testing to detect previous or recent infection with severe acute respiratory syndrome coronavirus (SARS-CoV)-2 can aid diagnosis. The interval between symptom onset and blood sampling affects the sensitivity and specificity of serologic tests [4]. The presence of antibodies was shown to be correlated with seroprotection in small animal models [5] and human cell lines, supporting SARS-CoV-2 antibody-based vaccine initiatives. It is currently unknown whether a positive antibody test indicates protective immunity against SARS-CoV-2 or how long humoral responses persist after natural infection.

The SARS-CoV-2 genome encodes four structural proteins including the spike glycoprotein and nucleocapsid, envelope, and membrane proteins. The nucleocapsid protein, one of the most abundant 
viral proteins, has a highly conserved amino acid sequence and high immunogenicity [6]. Tests that detect antibodies to SARS-CoV-2 nucleocapsid proteins are generally more sensitive than those targeting antibodies to spike glycoprotein $[7,8]$. Nucleocapsid protein plays a critical role in viral pathogenesis, including aggravation of lung injury by mannan-binding lectin-associated serine protease (MASP)-2mediated complement overactivation [9]. The protein has several epitopes that stimulate B and T cell responses and are suitable for vaccine formulations [10]. Moreover, as most SAR-CoV-2 vaccines used in humans are based on the spike antigen, the detection of antibody responses against nucleocapsid protein may be useful for distinguishing between serologic responses to infection and vaccination [11, 12].

The half-life of the SARS-CoV-2 antibody response in a cohort varies according to individuals' age, ethnicity, and symptom severity $[4,13]$. Immune responses against SARS-CoV-2 spike and nucleocapsid proteins are generally detectable within $1-3$ weeks after infection $[14,15]$. SARS-CoV-2 immunoglobulin ( $\mathrm{lg}) \mathrm{M}$ antibodies can be detected as early as 3-6 days after symptom onset while $\mathrm{IgG}$ antibodies can be detected after 8 days [16]. A study on the kinetics of the antibody response and disease severity in patients with SARS-CoV-2 infection found that more severe cases had higher levels of IgG [17]. Monitoring the persistence of anti-N IgG following natural infection is essential for estimating population immunity and guiding vaccination strategies.

This study evaluated the SARS-CoV-2 anti-N IgG seropositivity rate in a longitudinal cohort of recovered COVID-19 patients 3-12 months after symptom onset. The early antibody response at 0-3 months after infection was previously reported [18]. We also examined factors associated with the persistence of anti$\mathrm{N}$ IgG responses such as age, interval between symptom onset and blood sampling, and disease severity.

\section{Results}

\section{General characteristics of the study population}

Demographic data of 531 individual participants (single time point donors, $n=302$; multiple time point donors, $n=229$ ) are shown in Table 1 . The mean ( \pm standard deviation) age of the participants was 37.1 \pm 12.3 years (range: $2-82$ years) and the median age was 36 years. The male-to-female ratio was 1.03:1 (269:262). Participants were divided into two groups according to disease severity: those without pneumonia symptoms $(n=420)$ and those with pneumonia symptoms $(n=111)$. Individuals in the pneumonia group were older than those in the nonpneumonia/asymptomatic group $(P=0.02)$. 
Table 1

Demographic data of participants and specimens in this study

\begin{tabular}{|c|c|c|c|c|}
\hline \multirow[t]{3}{*}{ Participants } & \multirow[t]{3}{*}{ Characteristic } & \multicolumn{2}{|l|}{ Symptoms } & \multirow{3}{*}{$\begin{array}{l}\text { Chi-squared } \\
(P \text {-value })\end{array}$} \\
\hline & & $\begin{array}{l}\text { Without } \\
\text { pneumonia, }\end{array}$ & $\begin{array}{l}\text { With } \\
\text { pneumonia, }\end{array}$ & \\
\hline & & $N=420$ & $N=111$ & \\
\hline \multirow[t]{2}{*}{ Age, years } & Median age & 35 & 39 & \\
\hline & Mean age (SD) & $36.8(11.9)$ & $40.9(13.1)$ & \\
\hline \multirow[t]{5}{*}{ Age, years } & $<20(\%)$ & $11 / 420(2.6)$ & $1 / 111(1.0)$ & $9.6(0.02)$ \\
\hline & $20-39(\%)$ & $\begin{array}{l}253 / 420 \\
(60.2)\end{array}$ & $\begin{array}{l}56 / 111 \\
(50.5)\end{array}$ & \\
\hline & $40-59(\%)$ & $\begin{array}{l}132 / 420 \\
(31.4)\end{array}$ & $\begin{array}{l}42 / 111 \\
(37.8)\end{array}$ & \\
\hline & > $59(\%)$ & $17 / 420(4.0)$ & $\begin{array}{l}12 / 111 \\
(10.8)\end{array}$ & \\
\hline & Unknown (\%) & $7 / 420(1.7)$ & $0 / 111(0.0)$ & \\
\hline \multirow[t]{2}{*}{ Collection } & Single collection (\%) & $\begin{array}{l}245 / 420 \\
(58.3)\end{array}$ & $\begin{array}{l}56 / 111 \\
(50.5)\end{array}$ & \\
\hline & $\begin{array}{l}\text { Multiple collections } \\
(\%)\end{array}$ & $\begin{array}{l}175 / 420 \\
(41.7)\end{array}$ & $\begin{array}{l}55 / 111 \\
(49.5)\end{array}$ & \\
\hline \multirow[t]{2}{*}{ Sex } & Male (\%) & $\begin{array}{l}209 / 420 \\
(49.8)\end{array}$ & $\begin{array}{l}60 / 111 \\
(54.1)\end{array}$ & $1.1(0.3)$ \\
\hline & Female (\%) & $\begin{array}{l}211 / 420 \\
(50.2)\end{array}$ & $\begin{array}{l}51 / 111 \\
(45.9)\end{array}$ & \\
\hline \multirow[t]{3}{*}{ Specimens } & \multirow[t]{3}{*}{ Characteristic } & Symptoms & & \multirow{3}{*}{$\begin{array}{l}\text { Chi-squared } \\
\text { (P-value) }\end{array}$} \\
\hline & & $\begin{array}{l}\text { Without } \\
\text { pneumonia, }\end{array}$ & $\begin{array}{l}\text { With } \\
\text { pneumonia, }\end{array}$ & \\
\hline & & $N=420$ & $N=111$ & \\
\hline \multirow[t]{3}{*}{$\begin{array}{l}3 \text { Months post symptom } \\
\text { onset at collection }\end{array}$} & Seropositivity (\%) & $\begin{array}{l}260 / 300 \\
(86.7)\end{array}$ & $\begin{array}{l}68 / 75 \\
(90.7)\end{array}$ & $2.0(0.4)$ \\
\hline & $\begin{array}{l}\text { Median anti-N IgG } \\
\text { S/C index (IQR) }\end{array}$ & $\begin{array}{l}4.5(2.7- \\
6.1)\end{array}$ & $\begin{array}{l}5.7(3.9- \\
6.6)\end{array}$ & \\
\hline & GMT $(95 \% \mathrm{Cl})$ & $\begin{array}{l}3.0(2.6- \\
3.4)\end{array}$ & $\begin{array}{l}4.2(3.5- \\
5.1)\end{array}$ & \\
\hline
\end{tabular}

Abbreviations: $\mathrm{Cl}$, confidence interval; GMT, geometric mean titer; IgG, immunoglobulin G; IQR, interquartile range; SD, standard deviation. 


\begin{tabular}{|c|c|c|c|c|}
\hline \multirow[t]{3}{*}{ Participants } & \multirow[t]{3}{*}{ Characteristic } & \multicolumn{2}{|l|}{ Symptoms } & \multirow{3}{*}{$\begin{array}{l}\text { Chi-squared } \\
(P \text {-value })\end{array}$} \\
\hline & & $\begin{array}{l}\text { Without } \\
\text { pneumonia, }\end{array}$ & $\begin{array}{l}\text { With } \\
\text { pneumonia, }\end{array}$ & \\
\hline & & $N=420$ & $N=111$ & \\
\hline \multirow[t]{3}{*}{$\begin{array}{l}6 \text { Months post symptom } \\
\text { onset at collection }\end{array}$} & Seropositivity (\%) & $\begin{array}{l}61 / 188 \\
(32.4)\end{array}$ & $\begin{array}{l}33 / 53 \\
(62.3)\end{array}$ & $\begin{array}{l}18.3(< \\
0.01)\end{array}$ \\
\hline & $\begin{array}{l}\text { Median anti-N IgG } \\
\mathrm{S} / \mathrm{C} \text { index (IQR) }\end{array}$ & $\begin{array}{l}0.8(0.2- \\
1.9)\end{array}$ & $\begin{array}{l}1.9(0.7- \\
3.5)\end{array}$ & \\
\hline & GMT $(95 \% \mathrm{Cl})$ & $\begin{array}{l}0.6(0.5- \\
0.8)\end{array}$ & $\begin{array}{l}1.3(0.9- \\
1.9)\end{array}$ & \\
\hline \multirow[t]{3}{*}{$\begin{array}{l}9 \text { Months post symptom } \\
\text { onset at collection }\end{array}$} & Seropositivity (\%) & $\begin{array}{l}31 / 158 \\
(19.6)\end{array}$ & $\begin{array}{l}18 / 49 \\
(36.7)\end{array}$ & $6.3(0.04)$ \\
\hline & $\begin{array}{l}\text { Median anti-N IgG } \\
\mathrm{S} / \mathrm{C} \text { index (IQR) }\end{array}$ & $\begin{array}{l}0.5(0.1- \\
1.0)\end{array}$ & $1(0.5-2.5)$ & \\
\hline & GMT $(95 \% \mathrm{Cl})$ & $\begin{array}{l}0.4(0.3- \\
0.5)\end{array}$ & $\begin{array}{l}0.9(0.6- \\
1.3)\end{array}$ & \\
\hline \multirow[t]{3}{*}{$\begin{array}{l}12 \text { Months post symptom } \\
\text { onset at collection }\end{array}$} & Seropositivity (\%) & $\begin{array}{l}23 / 107 \\
(21.5)\end{array}$ & $\begin{array}{l}15 / 36 \\
(41.7)\end{array}$ & $6.3(0.04)$ \\
\hline & $\begin{array}{l}\text { Median anti-N IgG } \\
\text { S/C index (IQR) }\end{array}$ & $\begin{array}{l}0.4(0.1- \\
0.9)\end{array}$ & $\begin{array}{l}1.1(0.6- \\
2.3)^{-}\end{array}$ & \\
\hline & GMT $(95 \%$ Cl) & $\begin{array}{l}0.4(0.3- \\
0.5)\end{array}$ & $\begin{array}{l}1.0(0.6- \\
1.5)\end{array}$ & \\
\hline Total specimens & Positivity (\%) & $\begin{array}{l}375 / 753 \\
(49.8)\end{array}$ & $\begin{array}{l}134 / 213 \\
(62.9)\end{array}$ & $\begin{array}{l}32.1(< \\
0.01)\end{array}$ \\
\hline
\end{tabular}

\section{Long-term anti-N IgG seropositivity rates}

The seropositivity rate did not differ significantly between groups at 3 months after symptom onset or first SARS-CoV-2 detection (Fig. 2). However, at 6, 9, and 12 months the rate was significantly higher in the pneumonia group $(P<0.05)$. Anti-N IgG was detected in $87.5 \%$ of recovered COVID-19 patients at 3 months (328/375), in 38.6\% (93/241) at 6 months, in $23.7 \%$ (49/207) at 9 months, and in $26.6 \%(38 / 143)$ at 12 months. The anti-N IgG seropositivity rate was significantly lower at 6,9 , and 12 months than at 3 months $(P<0.01)$ and lower at 9 and 12 months than at 6 months $(P<0.01)$.

We compared the anti-N IgG sample/cutoff (S/C) index at 3, 6, 9, and 12 months after symptom onset or first SARS-CoV-2 infection (Fig. 3). The median IgG S/C index at 3 months was 4.8 (interquartile range [IQR]: 2.9-6.2) and decreased to 1.0 (IQR: 0.3-2.2) at 6 months, 0.6 (IQR: 0.2-1.3) at 9 months, and 0.6 (IQR: $0.2-1.5)$ at 12 months. 
When classified by disease severity, the anti-N IgG S/C index decreased over time in all patients. The median IgG S/C index at 3 months tended to be higher in the pneumonia group than in the nonpneumonia/asymptomatic group (5.7 [IQR: 3.9-6.6] vs 4.5 [IQR: 2.7-6.1]), although the difference did not reach statistical significance (Fig. 4). When classified by sex, there was no difference in anti-N IgG S/C index between males and females of the same disease severity $(P=0.69)$ (Fig. 5).

\section{Correlation analysis for anti-N IgG seropositivity}

Pearson product-moment correlation coefficients were computed to assess the relationship between IgG $\mathrm{S} / \mathrm{C}$ index and patient variables. The IgG S/C index was negatively correlated with interval between symptom onset and blood sampling $(r=-0.567, P<0.01)$ but positively correlated with age $(r=0.192, P<$ 0.01). The relationship between anti-N IgG S/C index and interval between symptom onset and blood sampling was plotted using the median and IQR. The regression analysis confirmed that IgG S/C index decreased over time. The patterns were similar in patients without $\left(r^{2}=0.321, P<0.01\right)$ and with $\left(r^{2}=\right.$ $0.321, P<0.01)$ pneumonia.

The anti-N IgG S/C index against SARS-CoV-2 in a longitudinal cohort of recovered COVID-19 patients who provided blood samples for at least three time points were plotted over time using median and IQR (Fig. 6). In total, there were 133 patients without pneumonia and 44 patients with pneumonia. The model predicted an anti-N IgG half-life of 75.4 days (95\% confidence interval $\left.[95 \% \mathrm{Cl}]=51.7-112.0, \mathrm{R}^{2}=0.37\right)$ in the non-pneumonia group and 107.6 days $\left(95 \% \mathrm{Cl}=39.4-974.5, \mathrm{R}^{2}=0.24\right)$ in the pneumonia group. No significant differences were observed in the decay kinetics between the two groups.

\section{Discussion}

Knowledge of the durability of the immune response against SARS-CoV-2 is essential for predicting protection and herd immunity and interpreting serology and epidemiology data. The present study evaluated the seropositivity rate of anti-N IgG against SARS-CoV-2 in patients 3-12 months after the onset of COVID-19 symptoms. The results showed that $>60 \%$ of patients who had recovered from COVID19 lost their detectable anti-N IgG at 6 months after symptom onset; at 9 and 12 months after natural infection, approximately one-quarter had detectable anti-N IgG.

Antibodies specific to the nucleocapsid protein of SARS-CoV-2 do not neutralize the virus but may still contribute to the immune control of infection through viral clearance by antibody-dependent cellular cytotoxicity [19]. The SARS nucleocapsid protein contains several T cell epitopes that stimulate the T cell response in a vaccine setting, inducing SARS-specific T cell proliferation and cytotoxic activity [20, 21]. A longitudinal study on the persistence of IgG to SARS-CoV-2 nucleocapsid protein detected using quantitative assays showed that anti-N IgG titer declined a few months after symptom onset, which occurred more rapidly in younger adults and asymptomatic individuals [13]. However, a qualitative study that used optical density as a measure showed that $90 \%$ of patients still had detectable ant-N IgG 1 year 
after symptom onset [22]. Although our study used a qualitative measure (IgG S/C index), our results are in agreement with those obtained with the quantitative assay.

In the present study, COVID-19 patients in the pneumonia group tended to generate a higher antibody response than those in the non-pneumonia/asymptomatic group; seropositivity rates also remained significantly higher in the former at 6, 9, and 12 months after infection. Our findings are in agreement with a previous study which demonstrated that anti-N IgG was significantly higher and persisted longer in patients with severe conditions $[23,24]$.

The anti-N IgG S/C index declined over time with an approximate half-life of 75.4 days in the nonpneumonia/asymptomatic group and 107.6 days in the pneumonia group. We calculated half-life from the anti-N IgG S/C index between days after symptom onset in a longitudinal cohort of recovered COVID19 patients who provided blood samples for at least three time points. The half-life of anti-N IgG was previously estimated as 68 and 71 days [25]. The half-life of antibodies is longer when assessed after as compared to before 3 months post symptom onset [25]. This is likely due to the rapid decline of antibody titer during the first few months post infection, after which the levels stabilize.

It was reported that persons who have had COVID-19 retained immune memory for at least 6 months [26]. Long-lived bone marrow plasma cells specific to SARS-CoV-2 have been detected in the bone marrow of recovered COVID-19 patients, serving as a persistent and essential source of antibodies [27]. A clinical study showed that reinfection was rare 1 year after primary infection, likely due to the protective effect of natural infection [28]. Nevertheless, there are limited data on whether protective immunity induced by natural infection with one strain of SARS-CoV-2 can confer cross-protection against variants. Because of the emergence of new variants and their circulation in the population, the Centers for Disease Control and Prevention recommends that the COVID-19 vaccine be given to recovered COVID-19 patients after 90 days post symptom onset. The World Health Organization has also stated that recovered COVID-19 patients can wait up to 6 months, during which time their natural immunity can protect them against reinfection.

This study had some limitations. Cross-sectional blood sampling may have prevented the detection of changes in immune response against SARS-CoV-2 compared to a longitudinal cohort. Additionally, analysis of other immune responses such as IgG against spike protein or spike receptor-binding domain, neutralizing antibodies, and memory $B$ cells, can provide insight into the humoral response following natural infection, which can guide immunization strategies for patients who have recovered from COVID19.

In summary, the results of this study demonstrate the generation of a persistent immunologic response to SARS-CoV-2 even after recovery from COVID-19; seropositivity was observed up to 12 months after symptom onset in one-quarter of previously infected patients. Additionally, the anti-N IgG S/C index was correlated with disease severity in patients. However, long-term monitoring is needed to determine whether this immunologic memory confers protection against reinfection with the same or a new variant of SARS-CoV-2. 


\section{Methods}

\section{Ethics statement}

The study protocol was approved by the Research Ethics Committee of the Faculty of Medicine, Chulalongkorn University (Institutional Review Board [IRB] no. 572/63). The IRB waived the requirement for informed consent because the samples were collected for routine preventive measures and were deidentified and anonymous. All methods were carried out according to relevant guidelines and regulations.

\section{Participants and blood sampling}

This cross-sectional and longitudinal cohort study enrolled patients diagnosed with COVID-19 by RT-PCR (The cobas SARS-CoV-2 Qualitative assay for use on the cobas $6800 / 8800$ Systems) between March 2020 and May 2021. There were 111 patients with pneumonia symptoms and 420 without these symptoms (non-pneumonia/asymptomatic group). Sample collection was performed $3 \pm 1,6 \pm 1,9 \pm 1$, and $12 \pm 1$ months after symptom onset or first detection of SARS-CoV-2 by RT-PCR in asymptomatic individuals. Patients' age, sex, symptom severity (ie, without or with pneumonia symptoms), and interval between symptom onset and date of blood sampling were recorded. A flow diagram of participant recruitment is shown in Fig. 1. A total of 302 participants provided blood samples at a single time point, while 229 provided multiple blood samples for 3-12 months. Symptom severity was determined from patients' medical records.

\section{Serologic testing}

Blood samples were centrifuged for $10 \mathrm{~min}$ at $2000 \mathrm{rpm}$ at room temperature. The supernatant (serum) was stored as aliquots in $2.0-\mathrm{ml}$ tubes at $-20^{\circ} \mathrm{C}$ until use. The specimens were tested for SARS-CoV-2 anti-N IgG by chemiluminescent microparticle immunoassay using the commercially available automated ARCHITECT system (Abbott Diagnostics, Sligo, Ireland), which calculates the mean chemiluminescent signal of a calibrator; the sample result is then divided by the stored calibrator result. The default unit for the SARS-CoV-2 anti-N IgG assay is the S/C index; $\mathrm{S} / \mathrm{C}$ values $\geq 1.4$ and $<1.4$ were defined as positive and negative, respectively, according to the manufacturer's instructions.

\section{Statistical analysis}

The anti-N IgG S/C index of samples was plotted against interval between symptom onset and blood sampling using individual data and median with IQR. Graphs were generated using Prism v9.0 software (Graph Pad, San Diego, CA, USA). Statistical analyses were performed using SPSS Statistics for Windows v21 software (IBM, Armonk, NY, USA). The chi-squared test was used to analyze the association between disease severity, interval between symptom onset and blood sampling, sex, and seropositivity rate. Pearson's correlation coefficient was computed to assess the relationship between the anti-N IgG S/C index and interval between symptom onset and blood sampling, age, and disease severity. The regression/correlation between the anti-N IgG S/C index and interval between symptom onset and blood sampling was calculated by linear regression. A $p$ value $<0.05$ was considered significant in all tests. 


\section{Declarations}

\section{Competing interests}

The authors declare no competing interests.

\section{Data availability}

The authors confirm that the data supporting the findings of this study are available within the article

\section{Funding}

This study was supported by a Research Chair Grant from the National Science and Technology Development Agency (P-15-50004), the Center of Excellence in Clinical Virology of Chulalongkorn University/King Chulalongkorn Memorial Hospital (GCE 59-009-30-005), and the Second Century Fund (C2F), Chulalongkorn University to Jira Chansaenroj.

\section{Author contributions}

J.C. drafted the manuscript; J.C., R.Y., N.P., and J.P. analyzed the data, prepared figures, and interpreted the results; M.S., P.C., S.J., P.K., J.S., C.S., O.T., T.P., C.B., D.I., D.C., M.I., R.K., A.M., and P.N. collected specimens; J.C., N.W., N.S., C.C., R.K., A.M., P.N., and Y.P. designed the study; N.W., N.S., C.C. and Y.P. revised the manuscript. All authors reviewed the manuscript, provided critical feedback, and approved the final draft.

\section{Acknowledgments}

We thank all staff from the Center of Excellence in Clinical Virology, Faculty of Medicine, Chulalongkorn University for their help with the testing.

\section{References}

1 Guan, W. J. et al. Clinical Characteristics of Coronavirus Disease 2019 in China. N Engl J Med 382, 1708-1720, doi:10.1056/NEJMoa2002032 (2020).

2 Wu, Z. \& McGoogan, J. M. Characteristics of and Important Lessons From the Coronavirus Disease 2019 (COVID-19) Outbreak in China: Summary of a Report of 72314 Cases From the Chinese Center for Disease Control and Prevention. JAMA 323, 1239-1242, doi:10.1001/jama.2020.2648 (2020).

3 Huang, C. et al. Clinical features of patients infected with 2019 novel coronavirus in Wuhan, China. Lancet 395, 497-506, doi:10.1016/S0140-6736(20)30183-5 (2020).

4 Long, Q. X. et al. Clinical and immunological assessment of asymptomatic SARS-CoV-2 infections. Nat Med 26, 1200-1204, doi:10.1038/s41591-020-0965-6 (2020). 
5 Rogers, T. F. et al. Isolation of potent SARS-CoV-2 neutralizing antibodies and protection from disease in a small animal model. Science 369, 956-963, doi:10.1126/science.abc7520 (2020).

6 Tilocca, B. et al. Comparative computational analysis of SARS-CoV-2 nucleocapsid protein epitopes in taxonomically related coronaviruses. Microbes Infect 22, 188-194, doi:10.1016/j.micinf.2020.04.002 (2020).

7 Burbelo, P. D. et al. Sensitivity in Detection of Antibodies to Nucleocapsid and Spike Proteins of Severe Acute Respiratory Syndrome Coronavirus 2 in Patients With Coronavirus Disease 2019. J Infect Dis 222, 206-213, doi:10.1093/infdis/jiaa273 (2020).

8 Caini, S. et al. Meta-analysis of diagnostic performance of serological tests for SARS-CoV-2 antibodies up to 25 April 2020 and public health implications. Euro Surveill 25, doi:10.2807/1560-

7917.ES.2020.25.23.2000980 (2020).

9 Flude, B. M. et al. Targeting the Complement Serine Protease MASP-2 as a Therapeutic Strategy for Coronavirus Infections. Viruses 13, doi:10.3390/v13020312 (2021).

10 Oliveira, S. C., de Magalhaes, M. T. Q. \& Homan, E. J. Immunoinformatic Analysis of SARS-CoV-2 Nucleocapsid Protein and Identification of COVID-19 Vaccine Targets. Front Immunol 11, 587615, doi:10.3389/fimmu.2020.587615 (2020).

11 Narasimhan, M. et al. Clinical evaluation of the Abbott Alinity SARS-CoV-2 spike-specific quantitative IgG and IgM assays among infected, recovered, and vaccinated groups. J Clin Microbiol, doi:10.1128/JCM.00388-21 (2021).

12 Bradley, T. et al. Antibody Responses after a Single Dose of SARS-CoV-2 mRNA Vaccine. N Engl J Med 384, 1959-1961, doi:10.1056/NEJMc2102051 (2021).

13 Lumley, S. F. et al. The duration, dynamics and determinants of SARS-CoV-2 antibody responses in individual healthcare workers. Clin Infect Dis, doi:10.1093/cid/ciab004 (2021).

14 Long, Q. X. et al. Antibody responses to SARS-CoV-2 in patients with COVID-19. Nat Med 26, 845-848, doi:10.1038/s41591-020-0897-1 (2020).

15 To, K. K. et al. Temporal profiles of viral load in posterior oropharyngeal saliva samples and serum antibody responses during infection by SARS-CoV-2: an observational cohort study. Lancet Infect Dis 20, 565-574, doi:10.1016/S1473-3099(20)30196-1 (2020).

$16 \mathrm{Li}$, Z. et al. Development and clinical application of a rapid IgM-IgG combined antibody test for SARSCoV-2 infection diagnosis. J Med Virol 92, 1518-1524, doi:10.1002/jmv.25727 (2020).

17 Luo, H. et al. The Characterization of Disease Severity Associated IgG Subclasses Response in COVID19 Patients. Front Immunol 12, 632814, doi:10.3389/fimmu.2021.632814 (2021). 
18 Chansaenroj, J. et al. Detection of SARS-CoV-2-specific antibodies via rapid diagnostic immunoassays in COVID-19 patients. Virol J 18, 52, doi:10.1186/s12985-021-01530-2 (2021).

19 Tso, F. Y. et al. Presence of antibody-dependent cellular cytotoxicity (ADCC) against SARS-CoV-2 in COVID-19 plasma. PLoS One 16, e0247640, doi:10.1371/journal.pone.0247640 (2021).

$20 \mathrm{Gao}$, W. et al. Effects of a SARS-associated coronavirus vaccine in monkeys. Lancet 362, 1895-1896, doi:10.1016/S0140-6736(03)14962-8 (2003).

21 Okada, M. et al. The development of vaccines against SARS corona virus in mice and SCID-PBL/hu mice. Vaccine 23, 2269-2272, doi:10.1016/j.vaccine.2005.01.036 (2005).

22 Yao, L. et al. Persistence of Antibody and Cellular Immune Responses in COVID-19 patients over Nine Months after Infection. J Infect Dis, doi:10.1093/infdis/jiab255 (2021).

23 Feng, X. et al. Longitudinal Profiling of Antibody Response in Patients With COVID-19 in a Tertiary Care Hospital in Beijing, China. Front Immunol 12, 614436, doi:10.3389/fimmu.2021.614436 (2021).

24 Lin, L. et al. Long-term infection of SARS-CoV-2 changed the body's immune status. Clin Immunol 218, 108524, doi:10.1016/j.clim.2020.108524 (2020).

25 Wheatley, A. K. et al. Evolution of immune responses to SARS-CoV-2 in mild-moderate COVID-19. Nat Commun 12, 1162, doi:10.1038/s41467-021-21444-5 (2021).

26 Dan, J. M. et al. Immunological memory to SARS-CoV-2 assessed for up to 8 months after infection. Science 371, doi:10.1126/science.abf4063 (2021).

27 Turner, J. S. et al. SARS-CoV-2 infection induces long-lived bone marrow plasma cells in humans. Nature, doi:10.1038/s41586-021-03647-4 (2021).

28 Vitale, J. et al. Assessment of SARS-CoV-2 Reinfection 1 Year After Primary Infection in a Population in Lombardy, Italy. JAMA Intern Med, doi:10.1001/jamainternmed.2021.2959 (2021).

\section{Figures}




\section{Participants}

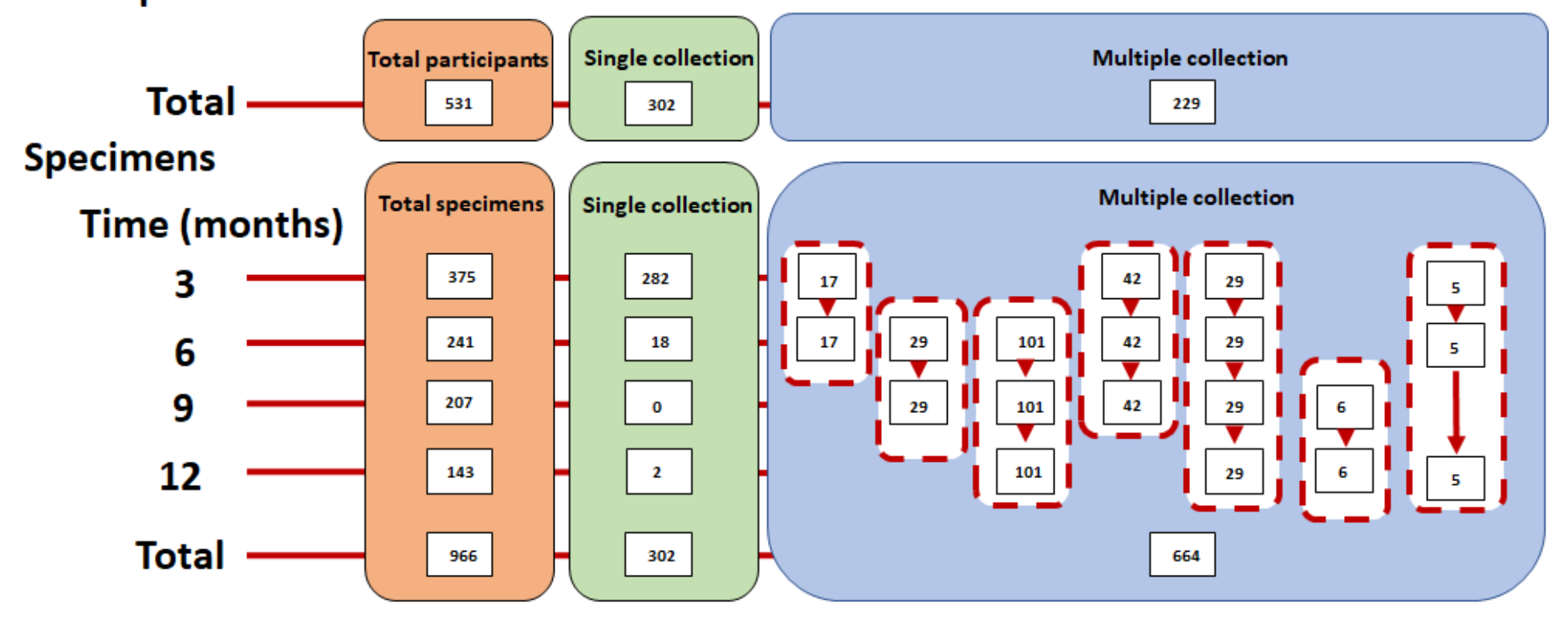

\section{Figure 1}

Flow diagram of participant recruitment and specimen collection in this study. A total of 531 participants were enrolled; 302 provided a blood sample at a single time point and 229 provided multiple samples. 


\section{\%Seropositive rate}

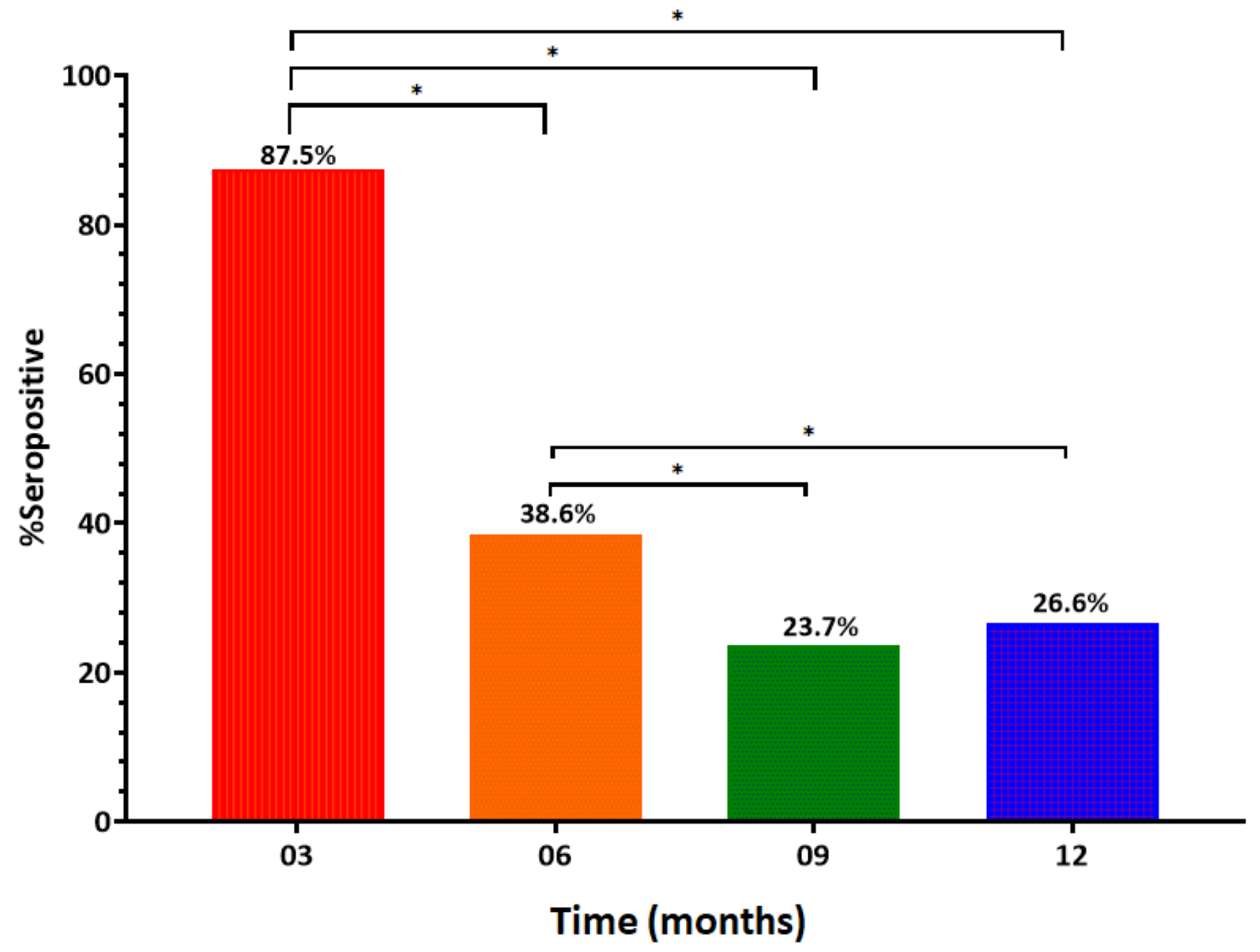

Figure 2

Seropositive rate among specimens at indicated time points after post symptom onset or first SARS-CoV2 detection. The cutoff was $1.4 \mathrm{~S} / \mathrm{C} ; \mathrm{S} / \mathrm{C} \geq 1.4$ was defined as positive and $\mathrm{S} / \mathrm{C}<1.4$ as negative. 


\section{Anti-Nucleocapsid IgG}

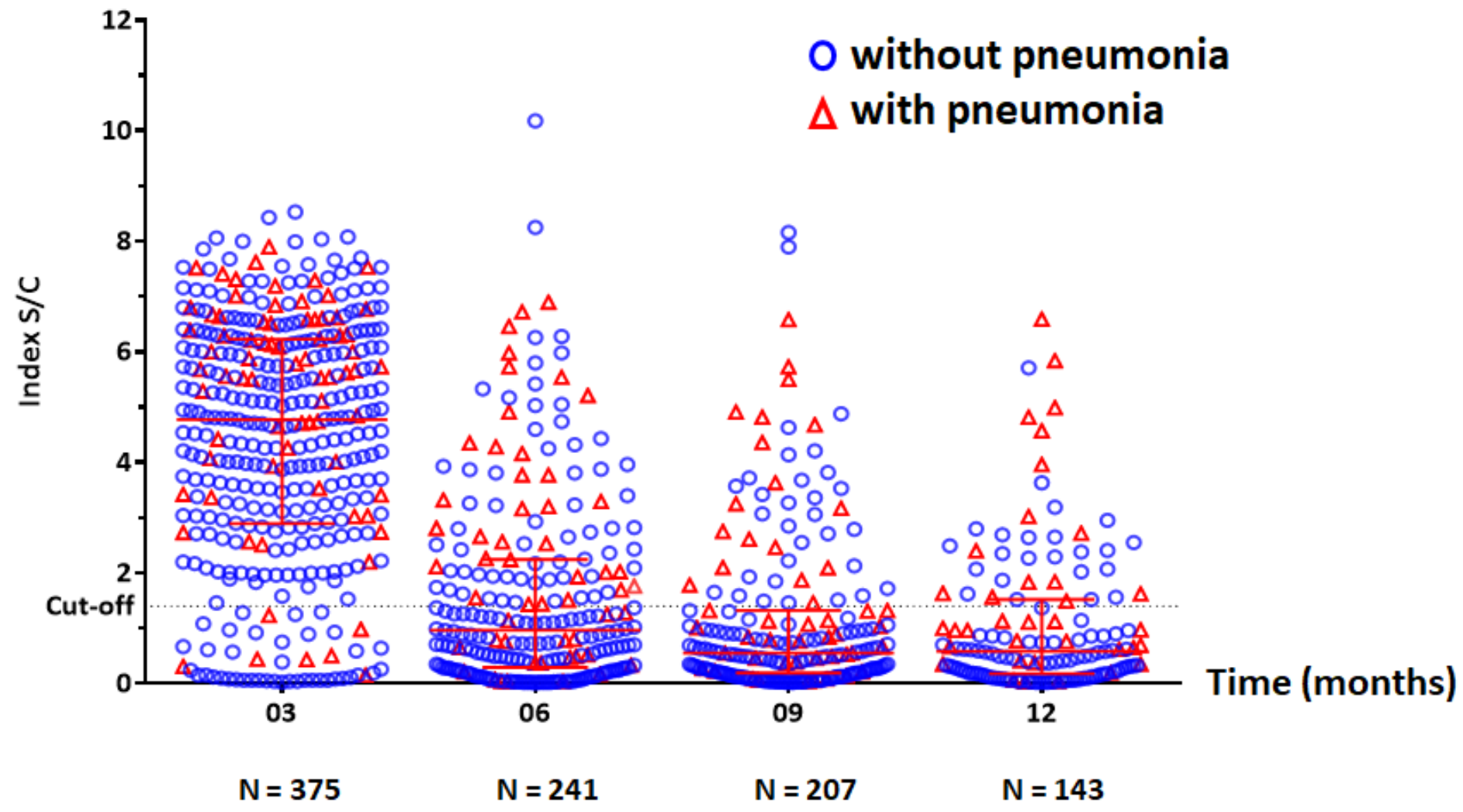

Figure 3

Scatter dot-plot with median and IQR showing the distribution of SARS-CoV-2-specific IgG anti-N S/C index in serum samples of COVID-19 patients with different disease severity at 3, 6, 9, and 12 months after symptom onset or first SARS-CoV-2 detection by RT-PCR. Red triangles and blue circles represent patients with and without pneumonia symptoms, respectively. 


\section{Anti-Nucleocapsid IgG}

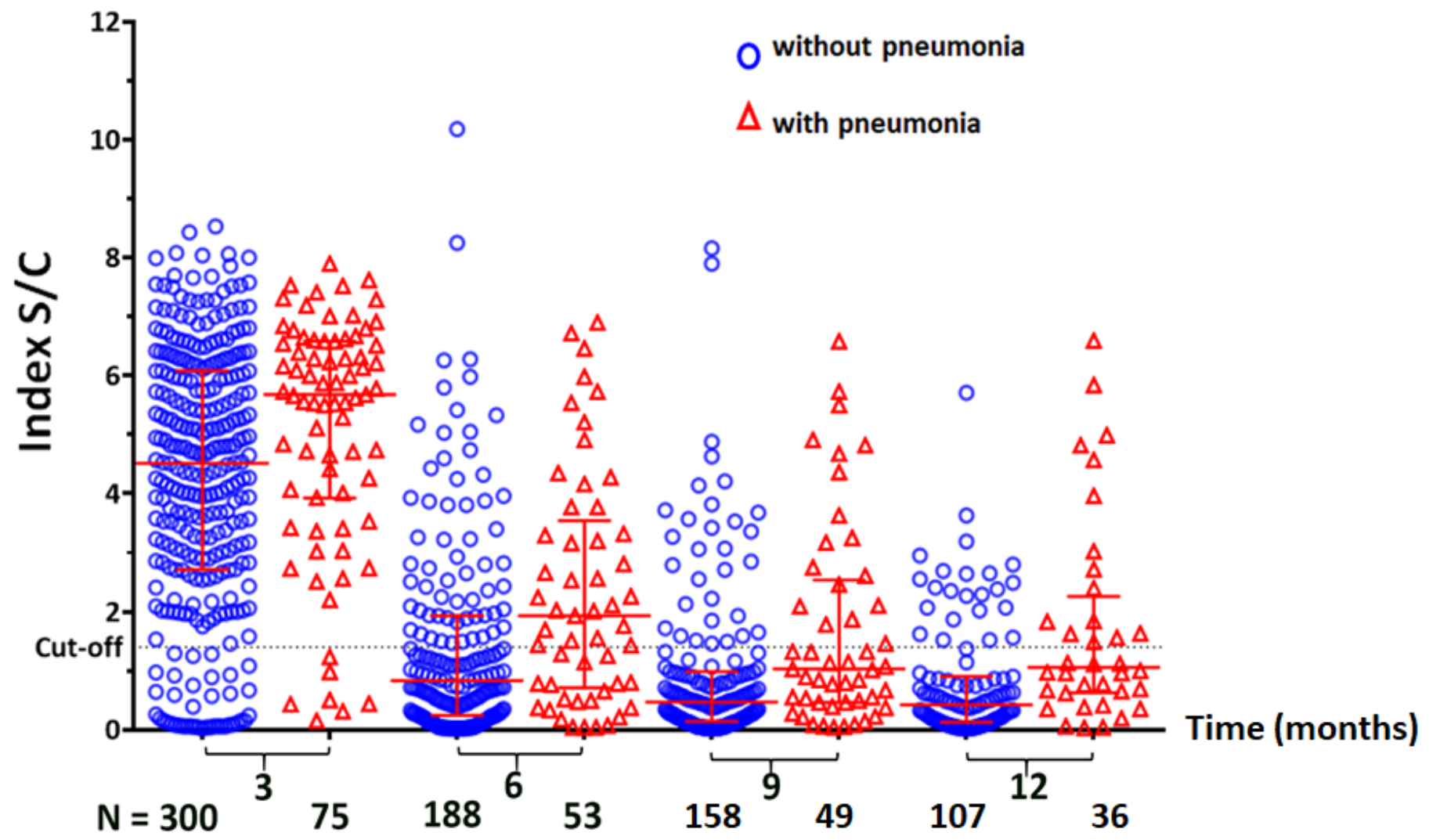

Figure 4

Scatter dot-plot of anti-N IgG S/C index in serum samples of COVID-19 patients with different disease severity. Red triangles and blue circles represent patients with and without pneumonia symptoms, respectively. 


\section{Anti-Nucleocapsid IgG}

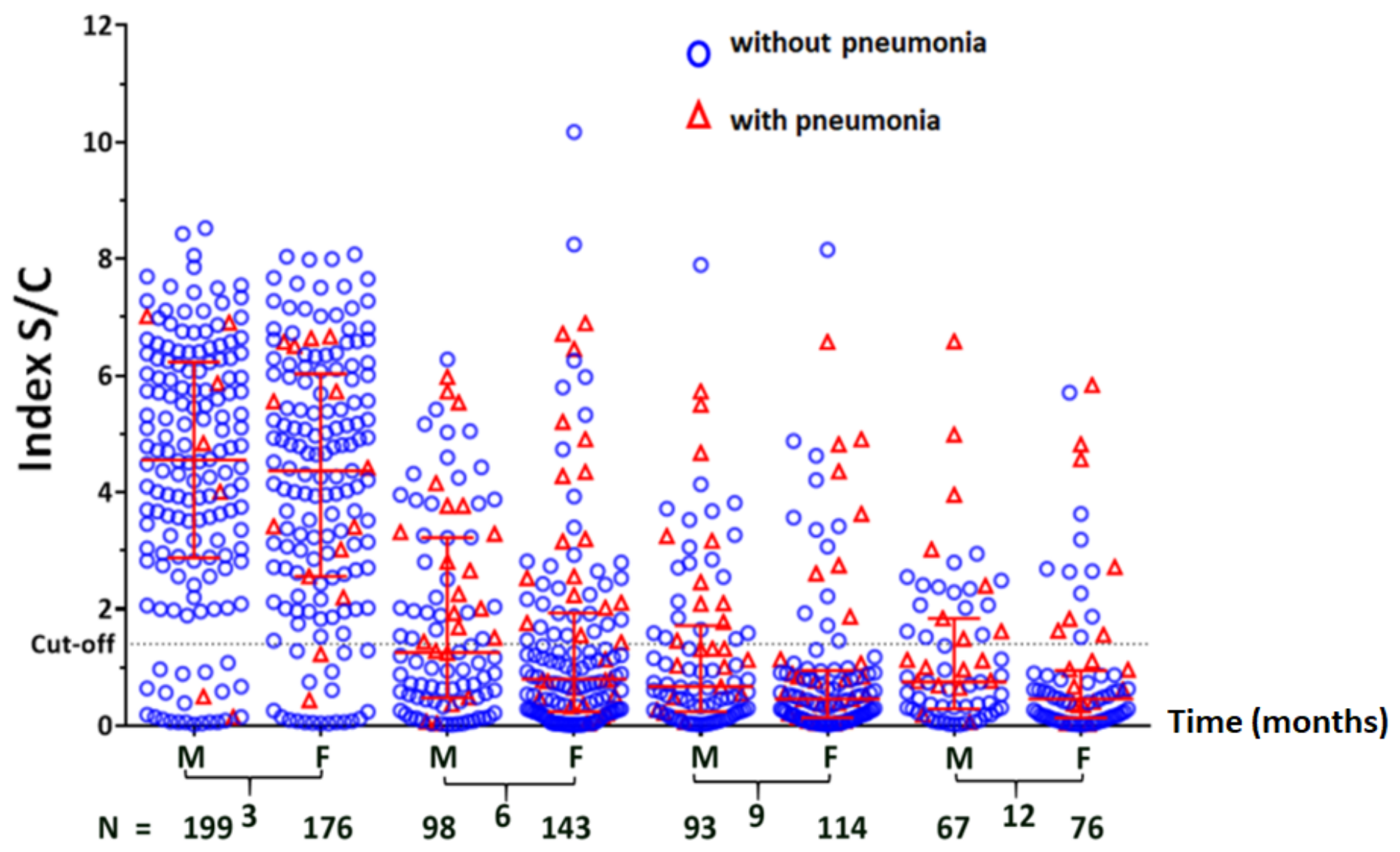

Figure 5

Scatter dot-plot classified by sex (F, female; M, male) with median and IQR showing the distribution of SARS-CoV-2-specific IgG anti-N S/C index in serum samples of COVID-19 patients with different disease severity. Red triangles and blue circles represent patients with and without pneumonia symptoms, respectively. 
Longitudinal without pneumonia

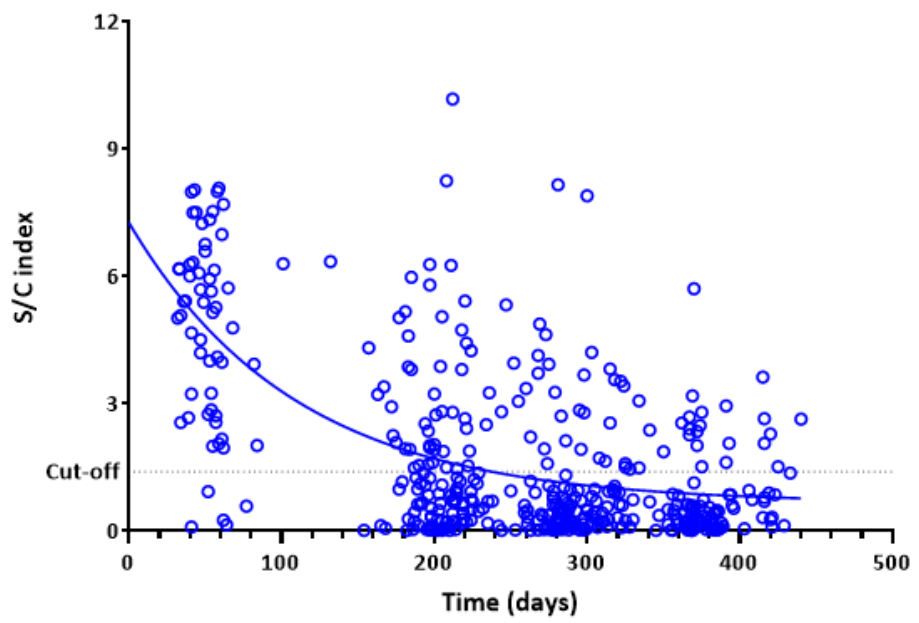

Longitudinal with pneumonia

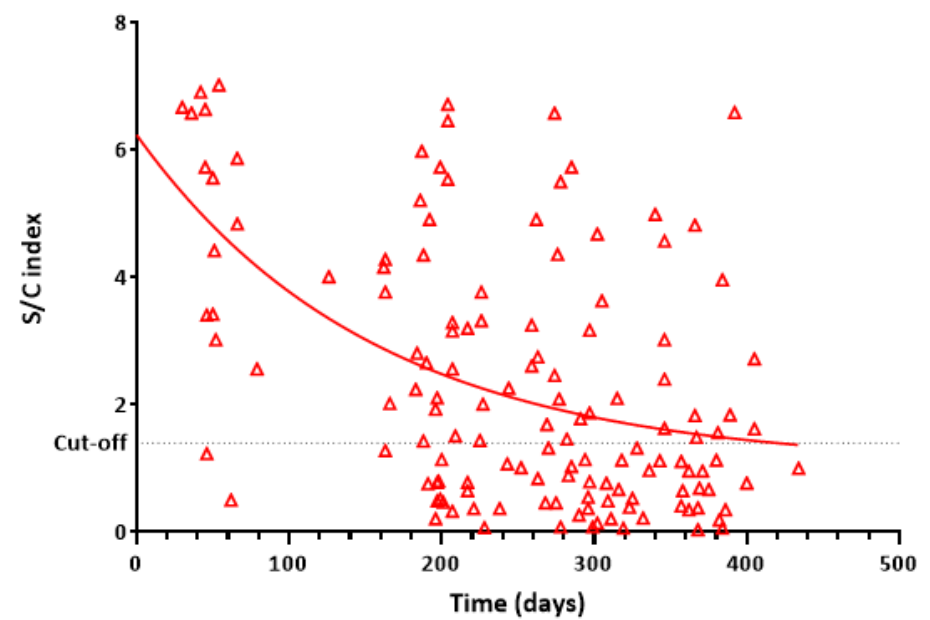

Figure 6

SARS-CoV-2-specific IgG anti-N S/C index in a longitudinal cohort of recovered COVID-19 patients who provided blood samples for at least three time points. a) Patients without pneumonia symptoms. b) Patients with pneumonia symptoms. 